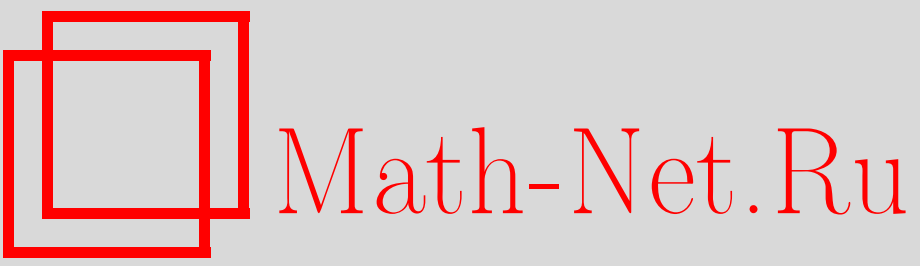

В. А. Артамонов, Тело рациональных квантовых функций, УМH, 1999, том 54, выпуск 4, 151-152

DOI: https://doi.org/10.4213/rm181

Использование Общероссийского математического портала Math-Net.Ru подразумевает, что вы прочитали и согласны с пользовательским соглашением

http://www.mathnet.ru/rus/agreement

Параметры загрузки:

IP : 54.172 .240 .79

26 апреля 2023 г., 15:25:34 


\title{
ТЕЛО РАЦИОНАЛЬНЫХ КВАНТОВЫХ ФУНКЦИЙ
}

\author{
В. А. Артамонов
}

Пусть $D$ - тело с заданным множеством автоморфизмов $\alpha_{1}, \ldots, \alpha_{n}, n \geqslant 2$. Предположим, что заданы также элементы $q_{i j} \in D^{*}, i, j=1, \ldots, n$, удовлетворяюшие условиям

$$
\begin{gathered}
q_{i i}=q_{i j} q_{j i}=Q_{i j r} Q_{j r i} Q_{r i j}=1, \quad \alpha_{i}\left(\alpha_{j}(d)\right)=q_{i j} \alpha_{j}\left(\alpha_{i}(d)\right) q_{j i}, \\
\text { где } Q_{i j r}=q_{i j} \alpha_{j}\left(q_{i r}\right) \text { и } d \in D . \text { Положим } \\
Q=\left(q_{i j}\right) \in \operatorname{Mat}(n, D) \text { и } \alpha=\left(\alpha_{1}, \ldots, \alpha_{n}\right) .
\end{gathered}
$$

Элементы $q_{i j}$ матрицы $Q$ называются системой мультипараметров. Обозначим через

$$
\Lambda=D_{Q, \alpha}\left[X_{1}, \ldots, X_{n}\right]
$$

ассоциативное кольцо, порожденное элементами тела $D$ и элементами

$$
X_{1}, \ldots, X_{n}
$$

с определяющими соотношениями

$$
\begin{gathered}
X_{i} d=\alpha_{i}(d) X_{i}, \quad d \in D, \quad i=1, \ldots, n \\
X_{i} X_{j}=q_{i j} X_{j} X_{i}, \quad 1 \leqslant i, j \leqslant n .
\end{gathered}
$$

Кольо (2) называется кольцом квантовых многочленов. В случае, когда $D$ - поле с тождественными автоморфизмами $\alpha_{1}, \ldots, \alpha_{n}$, алгебра $\Lambda$ возникает в некоммутативной алгебраической геометрии как координатное кольцо $\mathscr{O}_{Q}\left(\mathbb{A}^{n}\right)$ квантового аффинного пространства $\mathbb{A}^{n}$ размерности $n$ [1]. Обзор результатов по квантовым многочленам можно найти в [2].

В работах [3]-[5] рассматривается тело частных $k_{q}(X, Y)$ алгебры $k_{q}[X, Y]$, где $q \in k^{*}$ не является корнем из 1 . Алгебра $k_{q}[X, Y]$ является частным случаем кольца $\Lambda$, когда $D=k$-поле с тождественными автоморфизмами $\alpha_{1}, \alpha_{2}$ и $q=q_{12}$. В этих работах обобщаются результаты В.И. Исковских [6] о строении группы автоморфизмов поля рациональных функций $k(X, Y)$ на случай тела $k_{q}(X, Y)$. Кроме того, в [5] показано, что централизатор любого элемента из $k_{q}(X, Y) \backslash k$ коммутативен и поэтому является подполем в $k_{q}(X, Y)$. В [4] изучаются инварианты конечных групп автоморфизмов тела $k_{q}(X, Y)$ и связи с квантовыми алгебрами Вейля $A_{1}^{q}(k)$.

Кольцо $\Lambda$ из (1) является нётеровой слева и справа областью с телом частных

$$
F=D_{Q, \alpha}\left(X_{1}, \ldots, X_{n}\right) .
$$

Обозначим через $\mathscr{Z}$ множество $\mathbb{Z}^{n}$ с дополнительным элементом $\infty$. Тогда $\mathscr{Z}$ является аддитивным абелевым моноидом, в котором $a+\infty=\infty$ для всех $a \in \mathscr{Z}$. Лексикографический порядок в $\mathbb{Z}^{n}$ продолжается в $\mathscr{Z}$, причем $a<\infty$ для всех $a \in \mathbb{Z}^{n}$. Зададим нормирование $f \mapsto\|f\|$ мультипликативной полугруппе кольца $\Lambda$ со значением в аддитивном абелевом моноиде $\mathscr{Z}$ следующим образом. Пусть $f \in \Lambda$ - ненулевой многочлен с младшим одночленом

$$
\mu X_{1}^{l_{1}} \cdots X_{n}^{l_{n}}, \quad \mu \in D^{*}, \quad l_{1}, \ldots, l_{n} \geqslant 0
$$

в смысле лексикографического порядка в $\mathbb{Z}^{n}$. Полагаем $\|f\|=\left(l_{1}, \ldots, l_{n}\right) \in \mathbb{Z}^{n}$. Если $f=0$, то полагаем $\|f\|=\infty$. Это нормирование продолжается до нормирования $F=$ $D_{Q, \alpha}\left(X_{1}, \ldots, X_{n}\right) \rightarrow \mathscr{Z}$, именно, если $f, g \in \Lambda \backslash 0$, то

$$
\left\|f^{-1} g\right\|=-\|f\|+\|g\|, \text { и }\|0\|=\infty .
$$

Приведем мальцевскую конструкцию тела $\mathscr{F}$ квантовых лорановских рядов [7]. Обозначим через $\mathscr{F}$ множество, состоящее из нуля и всех формальных рядов

$$
f=\sum_{l \in \mathbb{Z}^{n}} f(l) X_{l}
$$

где $X^{l}=X_{1}^{l_{1}} \cdots X_{n}^{l_{n}}$, если $l=\left(l_{1}, \ldots, l_{n}\right) \in \mathbb{Z}^{n}$. При этом предполагается, что носитель $\operatorname{supp} f$, т.е. множество всех таких $l \in \mathbb{Z}^{n}$, что $f(l) \neq 0$, является артиновым в смысле лексикографического порядка в $\mathbb{Z}^{n}$. Множество $\mathscr{F}$ является кольцом относительно покоэффициентного сложения и относительно умножения, индуцированного умножением одночленов в соответствии с (2).

Работа частично поддержана грантом INTAS 93-436, грантами Российского фонда фундаментальных исследований 99-01-00382, 96-15-96050, программой “Университеты России”-5527. 
ТеОРемА 1. $\mathscr{F}$ является телом с нормированием

$$
\|f\|= \begin{cases}\min (\operatorname{supp} f) \in \mathscr{Z}, & \text { если } f \neq 0 \\ 0 \in \mathscr{Z}, & \text { если } f=0\end{cases}
$$

Существует вложение колещ $\phi: F \rightarrow \mathscr{F}$, сохраняющее нормирования.

ОПредЕЛЕниЕ 2. Пусть $N$ - подгруппа в мультипликативной группе $D^{*}$ тела $D$, порожденная коммутантом $\left[D^{*}, D^{*}\right]$ и всеми элементами вида $z^{-1} \alpha_{i}(z)$, где $z \in D^{*}$ и $i=1, \ldots, n$. Кольцо $\Lambda$ (тело $F$ ) является кольцом общих квантовых многочленов (телом общих рациональных квантовых функциц̆), если образы всех мультипараметров $q_{i j}, 1 \leqslant i<j \leqslant n$, независимы в мультипликативной группе $D^{*} / N$.

Всюду в дальнейшем предполагается, что $F$ - тело общих квантовых рациональных функций.

Теорема 3. Пусть $\gamma$ - автоморфизм тела $F$, действующий тождественно на D. Тогда существуют такие әлементы $\gamma_{1}, \ldots, \gamma_{n} \in D^{*}, u_{1}, \ldots, u_{n} \in F$ и такое челое число $\varepsilon= \pm 1$, что

(3) $\quad \gamma\left(X_{i}\right)=\gamma_{i} X_{i}^{\varepsilon}+u_{i}, \quad\left\|u_{i}\right\|>\varepsilon\left\|X_{i}\right\|, \quad i=1, \ldots, n$.

При этом отображение $\theta: F \rightarrow F$, при котором

$$
\theta\left(X_{i}\right)=\gamma_{i} X_{i}^{\varepsilon}, \quad \gamma_{i} \in D^{*}, \quad \varepsilon= \pm 1
$$

для любых $i=1, \ldots, n u \theta(d)=d$ для любого $d \in D$, является автоморфизмом тела $F$.

Теорема 4. Предположим, что каждый мультипараметр $q_{i j}, 1 \leqslant i, j \leqslant n$, лежит в чентре тела $D$ и $\alpha_{1}, \ldots, \alpha_{n}$ действуют тождественным образом на $D$. Пусть $\gamma-$ автоморфизм тела $F$, действующий тождественно на теле $D$ и имеющий вид (3) $\varepsilon=1$. Тогда существует такой әлемент $z \in \mathscr{F}$, что $\|z\|>0$ и $\gamma\left(X_{i}\right)=\gamma_{i}(1-z)^{-1} \times$ $X_{i}(1-z)$ для каждого $i=1, \ldots, n$.

Теорема 5. Предположим, что каждый мультипараметр $q_{i j}, 1 \leqslant i, j \leqslant n$, лежит в чентре тела $D$ и $\alpha_{1}, \ldots, \alpha_{n}$ действуют тождественным образом на $D$. Пусть $G$ конечная группа автоморфизмов тела $F$, действующих тождественно на теле $D$ u имеющих вид (3) с $\varepsilon=1$. Тогда группа $G$ абелева.

ТЕОрема 6. Предположим, что $D=k$ - поле нулевой характеристики с тождественными автоморфизмами $\alpha_{1}, \ldots, \alpha_{n}$. Пусть $\gamma$ - автоморфизм тела $F$, действующий тождественно на теле $D$ и имеющий вид (3) $с \varepsilon=1$. Тогда существует такой элемент $z \in \mathscr{F}$, ито $\|z\|>0$ и $\gamma=\operatorname{Ad}(1-z) \cdot \theta \cdot \operatorname{Ad}(1-z)^{-1}$, где $\operatorname{Ad}(1-w)$ - внутренний автоморфизм $\mathscr{F}$ сопряжсения с помощью $1-z$, т.е. $\operatorname{Ad}(1-z)(f)=(1-z) f(1-z)^{-1}$ для всех $f \in \mathscr{F}$.

Теорема 7. Пусть әлемент $f \in \mathscr{F}$ перестановочен $с X_{1}, \ldots, X_{n}$ с каждым әлементом из $D$. Тогда $f \in D$, причем $f$ является чентральным әлементом тела $\mathscr{F}$. $B$ частности, иентр тела $\mathscr{F}$ содержится в $D$.

Теорема 8. Пусть $D$ - поле нулевой характеристики и $\alpha_{1}, \ldots, \alpha_{n}$ тождественнь на D. Предположим, что $f \in \mathscr{F} \backslash D$. Тогда централизатор әлемента $f$ в $\mathscr{F}$ коммутативен.

\section{СПИСОК ЛИТЕРАТУРЫ}

[1] Демидов Е. Е. Квантовые группы. М.: Факториал, 1998. [2] Артамонов В. А. // УМH. 1998. T. 53. № 4. C. 3-76. [3] Alev J., Dumas F. // Collect. Math. 1995. V. 46. № 1-2. P. 1-9. [4] Alev J., Dumas F. // Comm. Algebra. 1997. V. 25. № 5. P. 1655-1672. [5] Artamonov V.A., Cohn P. M. On the skew field of rational functions on the quantum plane // Kurosh Algebraic Conference'98, Abstracts of Talks. Moscow: Moscow Univ., 1998. P. 28-29. [6] Исковских В. А. // Вестн. МГУ. Сер. 1. Матем., мех. 1983. № 5. С. 43-48. [7] Мальцев А. И. // Докл. АН СССР. 1948. Т. 60. С. 1499-1501.

Московский государственный

Принято редколлегией университет им. М. В. Ломоносова 21.07.1999

E-mail: artamon@mech.math.msu.su 